\title{
Photo-Identification of Humpback and Gray Whales Using Affine Moment Invariants
}

\author{
N. Kehtarnavaz ${ }^{1}$, V. Peddigari ${ }^{1}$, C. Chandan $^{1}$, W. Syed ${ }^{1}$, \\ G. Hillman ${ }^{2}$, and B. Wursig ${ }^{3}$ \\ ${ }^{1}$ Department of Electrical Engineering, University of Texas at Dallas \\ kehtareutdallas.edu \\ ${ }^{2}$ Department of Pharmacology, University of Texas Medical Branch at Galveston \\ ${ }^{3}$ Marine Biology Department, Texas A\&M University
}

\begin{abstract}
This paper discusses the identification of humpback and gray whales based on images of their flukes. White patches appearing on a fluke constitute unique identifying features which are used here to match a query fluke image against a database of fluke images. The fluke area is first extracted from a fluke image via the live-wire edge detection algorithm. Optimal thresholding is then applied to the fluke area to obtain the patches on the fluke automatically. Next, the affine moment invariants of patches are computed and stored in a feature database. Finally, the database images are ranked depending on the degree of similarity between the query and database invariants. The results obtained are presented in the form of a performance box plot showing the reduction in the amount of manual search for various percentages of test images.
\end{abstract}

\section{Introduction}

Individual identification of humpback and gray whales is of interest to marine mammalogists. Due to practical and legal obstacles associated with tagging the whales, the method of photo-identification offers an alternative identification approach. This approach relies on unique natural markings or features which are captured in photographs taken from research ships. White/light gray patches on the flukes of humpback whales and white/light gray blotches on the flukes of gray whales constitute relatively unique natural markings and are thus used here for whale identification. Figure 1 shows a sample fluke image of a humpback and a gray whale.

In the literature, one can find WhaleNet [3] as an identification approach for humpback whales. WhaleNet is a graphical-user-interface program that allows one to narrow down the search by visually selecting one of 18 possible fluke types. In [1], the curve matching technique, which we originally developed for identification of bottlenose dolphins [2], was applied to fluke edges of humpback whales. This curve matching technique consists of a string representation scheme describing curves at both syntactic (structure) and semantic (attribute) levels, and a string matching strategy.

\footnotetext{
* This research was supported by the Grant DBI-0077661 from the National Science Foundation.
} 
In this paper, we have developed a patch-matching technique to narrow down the manual search process by using the relatively unique identifying patches present on the fluke surface of the animals. This computer-assisted technique can be used as a follow-up identification phase to WhaleNet after specifying the fluke type. It can also be utilized as a standalone system to identify an individual whale without specifying the fluke type. The developed technique is based on affine moment invariants as formulated by Flusser and Suk [4]. For identification, affine moment invariants of a query fluke patch are compared with a database of previously computed affine moment invariants. The database images are then ranked depending on the degree of similarity between invariants.

The developed fluke matching technique is discussed in more detail in section 2. A Matlab-based graphical-user-interface software, named PatchMatch, is also developed for users to easily select patches and perform identification. This interface software is briefly described in section 3 . The results and their analysis are presented in section 4 .

\section{Fluke Patch Identification}

The patch matching technique developed in this paper consists of a patch detection part, an affine moment invariant computation part, and a matching part. Figure 2 provides a block diagram of the different stages of the photo-identification process.

During training, affine moment invariants of a database of fluke patches are computed and stored in a feature database. During matching, the affine moment invariants of a query patch are computed and matched against the feature database in order to identify the best matching individuals. In case of humpback whales, the user may enter the fluke type as specified by WhaleNet in order to lower the database size.

\subsection{Patch Extraction}

The matching process requires the detection or extraction of patches from fluke images. Many approaches based on edge detection, region growing, and thresholding can be utilized to extract patches from fluke images. However, the outcomes of such approaches depend on either some user-defined parameters or a great deal of user interaction. Here we have deployed the live-wire edge detection algorithm, as described in [5], to define the fluke area. This algorithm performs edge detection by obtaining the optimal path in a weighted graph representing the fluke boundary. This algorithm has been successfully deployed for extraction of dorsal fin edges as part of our FinScan [6] software. In contrast to many edge detection algorithms, this algorithm has been found to be insensitive to various lighting conditions under which the images are taken.

Next, the patches are detected via the optimal thresholding algorithm described in [7]. This algorithm optimally divides the histogram of the fluke area into patch and non-patch areas.

\subsection{Affine Transformation}

Affine transformation is an important subgroup of the general class of projective transformations. It involves translation, rotation, scaling and possible skewing (out- 
of-plane rotation). Mathematically, for the two-dimensional case, it can be represented by the following equation:

$$
\left(\begin{array}{l}
u \\
v
\end{array}\right)=A_{2 \times 2}\left(\begin{array}{l}
x \\
y
\end{array}\right)+b_{2 x 1}
$$

where $u v^{t}$ represents the transformed coordinates of an original point $x y^{t}$, ma$\operatorname{trix} A$ represents rotation, scaling and skewing, while $b$ is a vector representing the translation effect. It is worth mentioning that it is necessary to consider invariance to affine transformations because the photos are taken not only from different distances but also with different degrees of skewness. Also, it is sufficient to consider invariance to affine transformations because fluke surfaces are almost planar and have dimensions smaller than the distance from the camera.

\subsection{Affine Moment Invariants}

Once the patches are detected, they are used as the input to an affine moment invariant (AMI) computation module. Figure 3 shows an example of the extracted patches for which the AMIs are computed. These invariants were derived by Flusser and Suk [4], improving on a previous attempt by $\mathrm{Hu}$ [8]. Flusser and Suk derived four affine moment invariants using second- and third-order moments. The expressions for these invariants are given below.

$$
\begin{gathered}
I_{1}=\left(\mu_{20} \mu_{02}-\mu_{11}{ }^{2}\right) / \mu_{00}{ }^{4} \\
I_{2}=\left(\mu_{30}{ }^{2} \mu_{03}{ }^{2}-6 \mu_{30} \mu_{21} \mu_{12} \mu_{03}+4 \mu_{30} \mu_{12}{ }^{3}+4 \mu_{21}{ }^{3} \mu_{03}-3 \mu_{21}{ }^{2} \mu_{12}{ }^{2}\right) / \mu_{00}{ }^{10} \\
I_{3}=\left(\mu_{20}\left(\mu_{21} \mu_{03}-\mu_{12}{ }^{2}\right)-\mu_{11}\left(\mu_{30} \mu_{03}-\mu_{21} \mu_{12}\right)+\mu_{02}\left(\mu_{30} \mu_{12}-\mu_{21}{ }^{2}\right)\right) / \mu_{00}{ }^{7} \\
I_{4}=\left(\mu_{20}{ }^{3} \mu_{03}{ }^{2}-6 \mu_{20}{ }^{2} \mu_{11} \mu_{12} \mu_{03}-6 \mu_{20}{ }^{2} \mu_{02} \mu_{21} \mu_{03}+9 \mu_{20}{ }^{2} \mu_{02} \mu_{12}{ }^{2}+12 \mu_{20} \mu_{11}{ }^{2} \mu_{21} \mu_{03}\right. \\
+6 \mu_{20} \mu_{11} \mu_{02} \mu_{30} \mu_{03}-18 \mu_{20} \mu_{11} \mu_{02} \mu_{21} \mu_{12}-8 \mu_{11}{ }^{3} \mu_{30} \mu_{03}-6 \mu_{20} \mu_{02}{ }^{2} \mu_{30} \mu_{12}+9 \mu_{20} \mu_{02}{ }^{2} \mu_{21}{ }^{2} \\
\left.+12 \mu_{11}{ }^{2} \mu_{02} \mu_{30} \mu_{12}-6 \mu_{11} \mu_{02} \mu_{30} \mu_{21}+\mu_{02} \mu_{30}\right) / \mu_{00}{ }^{2}
\end{gathered}
$$

where $\mu_{p q}$ denotes the $p q$ th central moment given by

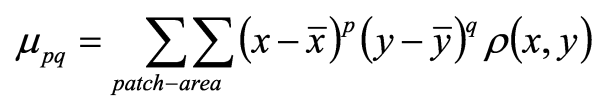

and $\bar{x}=m_{10} / m_{00}, \bar{y}=m_{01} / m_{00}, \quad(x, y)$ is a density function, $m_{p q}$ is the $p q$ th order moment given by

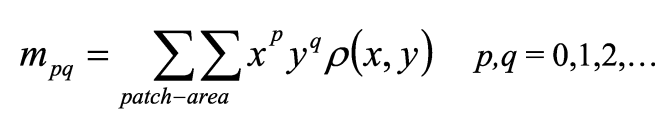

Due to the highly sensitive nature of patch intensity values to outdoor lighting conditions, the density function $(x, y)$ is considered to be a binary function here with 
value 1 assigned to patch areas and value 0 assigned to non-patch areas. The fluke area is divided into a left and a right half by using PatchMatch. The AMIs for each half are computed and entered as an 8-dimensional feature vector into a feature database. This process is repeated for all the images. The feature database is then used for the matching procedure, presented next.

\subsection{Matching}

The final part of the identification technique consists of matching the affine moment invariants of a query image against a database of affine moment invariants previously stored in the feature database. This is done by computing the Euclidean distance between the affine moment invariant vectors (for each corresponding fluke-halves), one vector corresponding to the invariants of the query image, and the other vector corresponding to the invariants of a database image. It should be mentioned that each invariant is normalized between -1 and 1 using its mean and variance in the database to avoid an invariant dominating the Euclidean distance.

\section{PatchMatch GUI}

The steps involved in the patch matching technique were incorporated into a graphical-user-interface (GUI) program, named PatchMatch, to allow efficient user interaction and processing. PatchMatch was written by utilizing the Matlab image processing and user interface toolboxes.

A popup menu gives the user the option to either train/set up a feature database, or match a query image against a feature database. Another popup menu allows the user to choose a sub-database containing images with the same fluke type. Upon matching, the images in the database are ranked depending on the ascending distances and then displayed 9 at a time for visual inspection by a mammologist. An example of the matching process is illustrated in Figure 4, where the first nine matched flukes are shown for a query image.

\section{Results and Discussion}

Two databases have been considered in this study. The first database comprises the humpback images provided on the Whalenet CD [3] distributed by the Wheelock College. The second database comprises the gray whale images collected by Wursig.

The WhaleNet database consists of a total of 911 fluke images. The software provided on the same $\mathrm{CD}$ allows the creation of 18 sub-databases corresponding to the 18 fluke types mentioned earlier. The number of images in the 18 sub-databases varies from 1 to 84 images. The half-submerged fluke images were excluded from our feature database due to their incomplete patch information. The remaining 461 unique images were thus used for the subsequent studies.

The gray whale database consists of 96 images taken from 38 individual gray whales. Despite the humpback database, this database includes 2 or 3 different images for each individual gray whale. One of the animals was excluded in our study as it had only one image of itself. 
For testing in case of humpback whales, the process of patch detection was repeated and the affine moment invariants were computed for each query image. A box plot was made to show the position of the first correct match for various percentages of the test images. Such plots exhibit performance of any identification system. Figure 5 shows the performance box plots for the humpback database studied. As indicated in this figure, more than $90 \%$ of the time the user needed to search through less than 50 images, instead of 461 images, to identify the right individual. In essence, the computer-assisted approach reduced the database to one-ninth of its size. Figure 5 also includes the comparison with the string curve matching method, reported previously for dorsal fins in [2], where the fluke edge information was used for matching. It is worth mentioning that better performance was obtained when using the sub-databases as specified by WhaleNet.

The ideal performance outcome is that, for any query or test image, the correct fluke is the very first image. However, in practice, there are many noise or distortion sources that cause deviation from the ideal outcome. Our analysis has revealed that the drop in performance from the ideal case is mostly due to the following factors: (a) ambiguity during patch detection caused by very low contrast patches and existence of very small patches, and (b) the finite resolution (limited number of pixels) of the images.

A study was carried out to examine the affine invariant property of affine moment invariants when the fluke surface is not parallel to the image plane during photography. This study revealed that very little loss in performance occurred for up to 60 degrees of out-of-plane rotation or skewness. Beyond 60 degrees, due to discretization and loss of image resolution, affine moment invariants started losing their effectiveness. In the database studied, however, it was observed that almost all the images had been taken with the fluke surface being more or less parallel (small amounts of skewness) with respect to the camera image plane.

For testing in case of gray whales, unlike humpback whales, the invariants of a query image were matched against all the other images in the database by leaving out the query image from the database, since this time there existed more than one image of an individual. As shown in Figure 6, it is seen that about $75 \%$ of the time the user needed to search through only one-fourth of the database to get a match to a different image of the query individual. Figure 6 also includes the comparison with the string curve matching method.

A study was carried out to find out how the performance changed with the number of blotches sorted in terms of their sizes. It was seen that the performance improved steadily when the number of blotches was increased to 10 . Thereafter, the performance remained more or less the same when considering up to 12 blotches and then started to degrade for higher number of blotches. This was due to the fact that the higher order blotches consisted of very few pixels and thus increased the identification sensitivity during the matching process.

\section{Conclusion}

In this paper, a computer-assisted photo-identification approach has been developed for the identification of humpback and gray whales. The obtained performance results indicate that in most cases this approach considerably reduces the number of images 
to be searched by a mammologist. For the databases studied, it was found that the correct match was seen in the first two sets of 9 displayed images $90 \%$ of the time in case of humpback whales and $75 \%$ of the time in case of gray whales.

\section{References}

1. Araabi, B.N.: Syntactic/Semantic Curve-Matching and Photo-Identification of Dolphins and Whales. PhD Dissertation, Texas A\&M University (2001)

2. Araabi, B.N., Kehtarnavaz, N., McKinney, T., Hillman, G., Wursig, B.: A String Matching Computer-Assisted System for Dolphin Photo-identification. Annals of Biomedical Engineering, Vol. 28. (2000), 1269-1279

3. WhaleNet - http://whale.wheelock.edu

4. Flusser, J., Suk, T.: Pattern Recognition by Affine Moment Invariants. Pattern Recognition, Vol. 26. (1993) 167-174

5. Mortensen, E., Barrett, W.: Interactive Segmentation with Intelligent Scissors. Graphical Models and Image Processing, Vol. 60. (1998) 349-384

6. Hillman, G., Wursig, B., Gailey, G., Kehtarnavaz, N., et. al: Finscan: a computer system for photographic identification of marine mammals. Proceedings of the $24^{\text {th }}$ EMBS International Conference, Houston (2002)

7. Otsu, N.: A Threshold Selection Method from Gray-Level Histogram. IEEE Transactions on System, Man, Cybernetics, Vol. SMC-9 (1979) 62-66

8. Hu, M.: Visual Pattern Recognition by Moment Invariants. IRE Trans. Inf. Theory, IT-8. (1962) 179-187

\section{Figures}
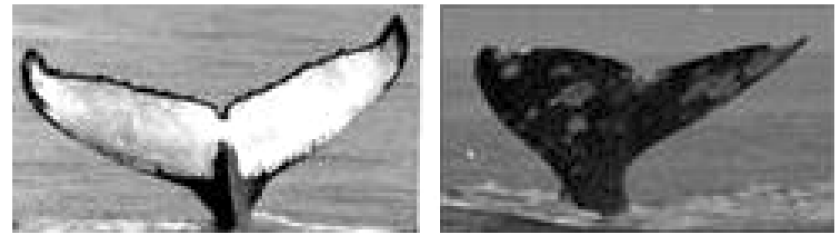

Fig. 1 - Sample fluke images of humpback whale (left) and gray whale (right)

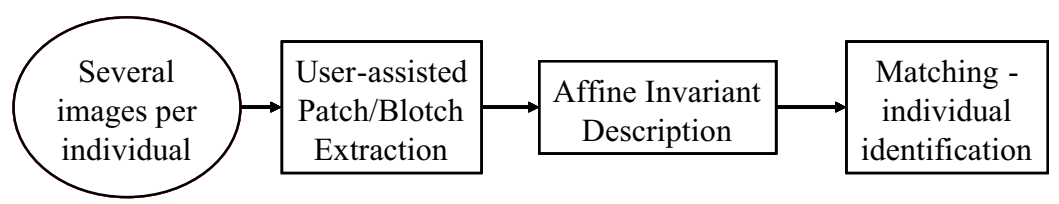

Fig. 2 - Block diagram of the photo-identification process 


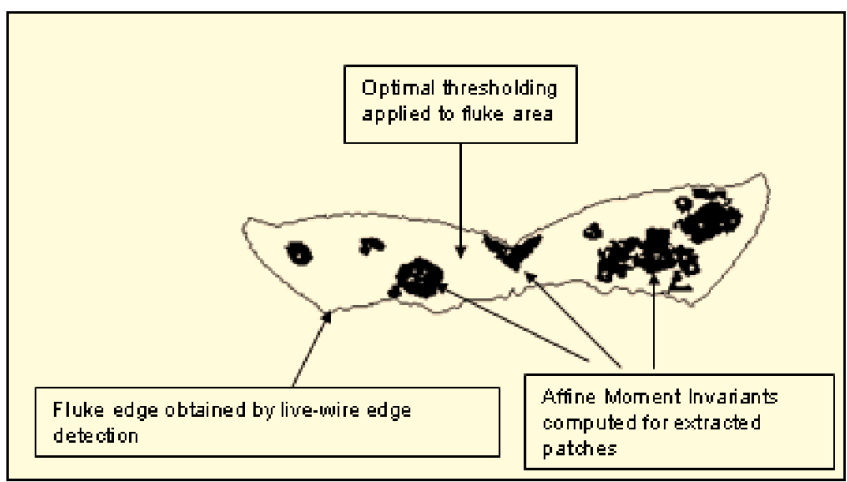

Fig. 3 - Edge detection and patch extraction for AMI computation

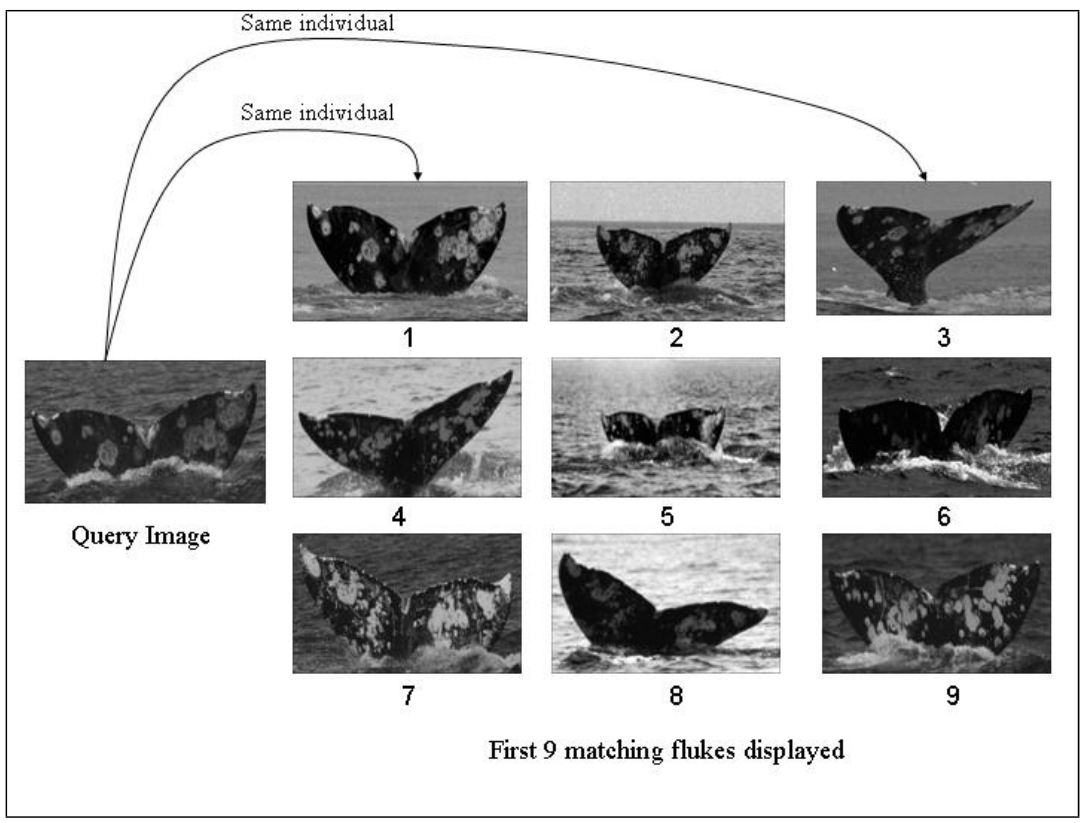

Fig. 4 - Identified matches for a query image 


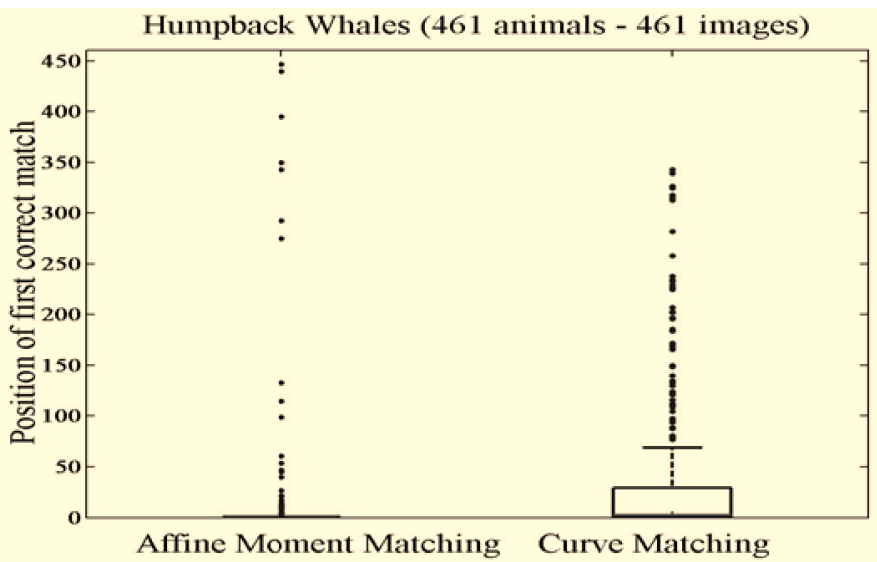

Fig. 5 - Box plots for humpback whales

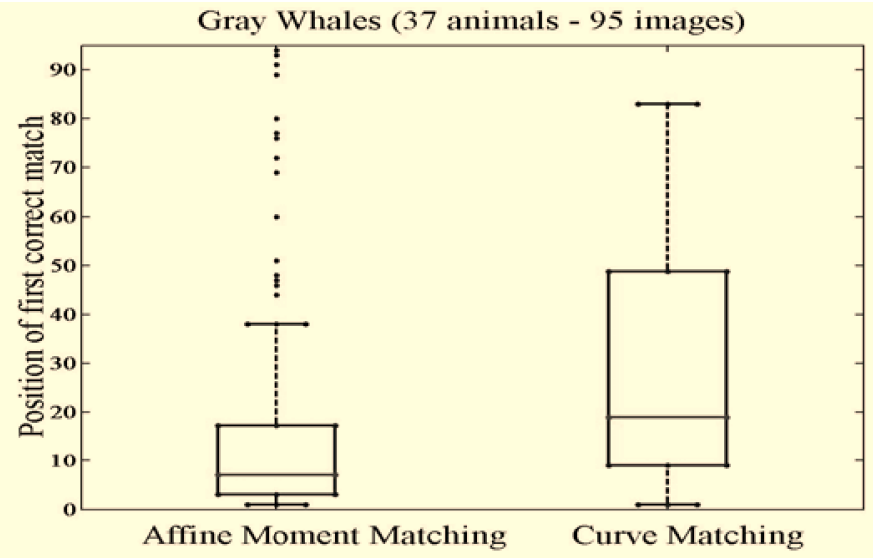

Fig. 6 - Box plots for gray whales 